\title{
La chirurgie en crise à l'ère de la COVID-19 : changements transformationnels en vue
}

\author{
David R. Urbach MD MSc, Danielle Martin MD MPP
}

Citation : CMAJ 2020 May 25;192:E585-6. doi : 10.1503/cmaj.200791-f; diffusion hâtive le 6 mai 2020

Entrevue avec l'auteur (en anglais) en baladodiffusion : soundcloud.com/cmajpodcasts/200791-com

Voir la version anglaise de l'article ici : www.cmaj.ca/lookup/doi/10.1503/cmaj.200791

$\mathbf{L}$ a fermeture des services en réponse à la pandémie de maladie à coronavirus 2019 (COVID-19) a mis sur pause les chirurgies non urgentes au Canada. On estime qu'il y a eu 45000 chirurgies de moins que le nombre attendu chez des adultes présentant des maladies non cancéreuses en Ontario à la fin mars et au début avril 2020, ce qui correspond à une réduction de plus de $90 \%$ comparativement à la même période de 4 semaines en $2019^{1}$. Le temps d'attente pour une chirurgie était déjà inacceptable bien avant la pandémie ${ }^{2}$. Il sera difficile de reprendre tout le retard accumulé, et nous aurons besoin d'un plan de rattrapage fondé sur des bases éthiques. Cependant, compte tenu du modèle actuel de prestation des soins chirurgicaux et de l'allocation des ressources mises à la disposition des chirurgiens dans les hôpitaux canadiens, il faudra s'attendre à des difficultés logistiques. Un modèle collaboratif à accès unique pourrait aider à régler le problème de façon efficiente, équitable et éthique.

Les modèles à accès unique abordent le problème du temps d'attente en dressant une liste qui oriente les patients vers le prochain professionnel disponible, en fonction du caractère urgent du cas et du degré de priorité. L'approche collaborative facilite la coordination des soins par la répartition des cas entre les différents membres de l'équipe : une intervention pourrait ne pas être effectuée par le premier chirurgien que le patient rencontre, mais plutôt par un autre membre de l'équipe aux compétences équivalentes qui connaît bien son cas.

Les modèles à accès unique s'inspirent de la théorie des files d'attente; ils sont déjà couramment utilisés dans l'industrie aéronautique, les services bancaires, les restaurants et les parcs d'attraction ${ }^{3,4}$, et ils bénéficient de l'appui des grandes organisations et commissions médicales nationales ${ }^{3}$. Chaque fois qu'ils ont été mis en place dans le milieu de la santé, les modèles de soins collaboratifs à accès unique ont été largement adoptés et appréciés des patients et des professionnels de la santé. Au Canada, ils ont été mis à l'essai en obstétrique, en chirurgie cardiaque, en arthroplastie, en transplantation, en oncochirurgie, en soins aigus et en chirurgie générale non urgente ${ }^{3,5,6}$. En outre, les médecins traitants préfèrent les modèles à accès unique, et de loin ${ }^{7}$. Les partisans de ces modèles soulignent leur efficacité pour la réduction et l'harmonisation du temps d'attente pour les consultations auprès des spécialistes et les chirurgies non urgentes².

\section{POINTS CLÉS}

- La pandémie de maladie à coronavirus 2019 (COVID-19) a profondément réduit la capacité des systèmes de santé à fournir les services non urgents prévus, tels que des chirurgies et d'autres interventions.

- Ensemble, les modèles collaboratifs à accès unique constituent une approche efficiente, juste et éthique pour rattraper le retard des chirurgies dans des circonstances où les ressources sont restreintes.

- Même après la pandémie de COVID-19, les modèles collaboratifs à accès unique demeureront des stratégies efficaces pour réduire le temps d'attente et améliorer l'expérience de soins des patients et les environnements de travail des chirurgiens.

Au Canada, la plupart des chirurgiens exercent seuls; ils acceptent les demandes de consultation des médecins traitants, puis s'occupent des soins de chaque patient pour l'entière période de prise en charge. Même si pour certains le lien personnel entre médecin traitant, chirurgien et patient est sacré, cette façon de faire est aléatoire, inefficace et inéquitable. Elle fait indûment varier le temps d'attente (un problème qui mine la confiance de la population envers les systèmes de santé au Canada) et place le chirurgien - plutôt que le patient - au centre de l'épisode de soins ${ }^{3}$.

Quand les services reprendront pour les chirurgies non urgentes, le retour à cette méthode ne sera pas acceptable. À court terme, la capacité de pratiquer les chirurgies prévues sera grandement limitée en raison des pressions fluctuantes exercées sur les hôpitaux soumis à des vagues récurrentes de COVID-19 et à l'obligation de gérer des problèmes d'effectifs, d'approvisionnement en équipement et en médicaments et de risque de transmission du virus. Des solutions créatives à la gestion de l'accumulation de demandes s'imposent. Les modèles à accès unique respectent les principes éthiques clés pour la distribution des ressources en période de restrictions, soit l'utilité, la justice, l'équité et l'équilibre, et ils offrent la souplesse nécessaire pour préserver l'autonomie des patients.

En temps normal, les soins collaboratifs sont jugés supérieurs, car ils permettent d'harmoniser la prise de décision, facilitent la préparation périopératoire et offrent la possibilité d'obtenir facilement une seconde opinion dans les cas complexes. À l'heure actuelle, en raison de l'épidémie de COVID-19, des chirurgiens opèrent 
les patients de leurs collègues dans les hôpitaux américains ${ }^{8}$. Si ces modèles de soins sont si efficients et efficaces, pourquoi ne sont-ils pas déjà répandus au Canada?

La principale raison est la réticence des chirurgiens et des autres professionnels de la santé à leur égard. Une bonne part de cette réticence provient de l'incertitude et de la peur, des émotions exacerbées par le stress occasionné par la crise sanitaire. Beaucoup croient que la façon actuelle de gérer les demandes de consultation fonctionne bien et permet d'orienter les patients vers les soins les meilleurs et les plus appropriés, et qu'une fois établi, le lien entre chirurgien et patient ne devrait jamais être perturbé. Les chirurgiens s'inquiètent aussi, et à raison, de leur autonomie professionnelle, de leur indépendance, de leur base de recommandations et de leur sécurité financière.

Mais les inquiétudes des chirurgiens ne devraient pas entraver les efforts pour trouver et adopter des stratégies novatrices permettant de se sortir de la crise de la COVID-19. Les inquiétudes soulevées par ces modèles sont légitimes, mais tout à fait gérables. Par exemple, les chirurgiens peuvent avoir l'impression que les patients n'accepteront personne d'autre que leur propre chirurgien. Or, il a été rapporté que la plupart des patients préfèrent réduire leur temps d'attente, même si cela signifie qu'ils seront pris en charge par le prochain professionnel disponible ${ }^{4}$. De plus, dans un modèle d'équipe de chirurgie, moins de la moitié des patients ont jugé important d'avoir le même chirurgien; les trois quarts se sont dits à l'aise de rencontrer leur chirurgien pour la première fois le jour de l'intervention ${ }^{6}$.

Même dans les meilleures circonstances, le changement est difficile. En 1906, à l'occasion de son Harveian Oration, livré au Royal College of Physicians of London, Sir William Osler a fait l'observation que «la douleur causée par une idée nouvelle est parmi les plus pénibles pour l'être humain ${ }^{9}$. En temps de crise, l'idée d'un changement de système peut sembler un obstacle insurmontable.

Il est donc important de se rappeler que les modèles collaboratifs à accès unique ne conviennent que pour les interventions courantes et standardisées que tous les chirurgiens de l'équipe peuvent accomplir avec brio. Même s'il y a une certaine variation quant à la qualité des soins prodigués par les différents professionnels, rien n'indique que le choix du chirurgien par le patient ou le médecin traitant est une bonne façon d'éviter les chirurgiens moins efficaces ${ }^{10,11}$. Ce sont les responsables cliniques, et non le public, qui devraient gérer les situations où les soins laissent à désirer. En fait, les modèles de soins collaboratifs facilitent la mise en évidence de variations dans la pratique et favorisent le soutien entre collègues dans le but d'améliorer la qualité et la fiabilité des soins et l'environnement de travail des chirurgiens ${ }^{12}$.

Au final, les chirurgiens veulent reprendre les opérations pour leurs patients. Plusieurs ont l'impression que leur carrière et leur gagne-pain sont menacés par la suspension des activités hospitalières imposées par la COVID-19. En donnant aux chirurgiens un accès égal aux blocs opératoires, les modèles collaboratifs à accès unique sont les mécanismes les plus équitables pour que tous les chirurgiens, sans égard au sexe, au nombre d'années d'exercice ou au réseau de recommandations existant, aient la possibilité de soigner leurs patients et de maintenir leurs compétences. Ces modèles de soin centrés sur les patients sont l'approche la plus équitable pour relever les énormes défis auxquels nous faisons face.
Les chirurgiens devraient joindre leur voix à celle des responsables des systèmes de santé et des hôpitaux et des décideurs pour réclamer l'intégration de cette approche au plan de reprise des chirurgies après la pandémie de COVID-19 et saisir l'occasion, qui ne se représentera pas de sitôt, de transformer considérablement les services chirurgicaux et de mettre en place un système de santé durable et éthique au Canada.

\section{Références}

1. Wait Time Information System (WTIS) portal. Toronto: Cancer Care Ontario. Accessible ici : https://www.ccohealth.ca/en/submit-data/wait-time-information -system-portal (consulté le 21 avril 2020).

2. Urbach DR. Improving access to health services in Canada. Healthc Manage Forum 2018;31:256-60.

3. Lopatina E, Damani Z, Bohm E, et al. Single-entry models (SEMs) for scheduled services: towards a roadmap for the implementation of recommended practices. Health Policy 2017;121:963-70.

4. Damani Z, Conner-Spady B, Nash T, et al. What is the influence of single-entry models on access to elective surgical procedures? A systematic review. BMJ Open 2017;7:e012225.

5. Zwiep TM, Greenberg JA, Balaa F, et al. Impact of group practices on patients, physicians and healthcare systems: protocol for a scoping review. BMJ Open 2018;8:e022164.

6. van den Heuvel B, Vair B, Porter G, et al. Patient compliance with a group model of care: the hernia clinic. Can J Surg 2012;55:259-63.

7. Ramchandani M, Mirza S, Sharma A, et al. Pooled cataract waiting lists: views of hospital consultants, general practitioners and patients. JR Soc Med 2002;95:598-600.

8. Lancaster EM, Sosa JA, Sammann A, et al. Rapid response of an academic surgical department to the COVID-19 pandemic: implications for patients, surgeons, and the community. J Am Coll Surg le 9 avril 2020 [Cyberpublication avant impression]. pii: S1072-7515(20)30312-4. doi: 10.1016/j.jamcollsurg.2020.04.007.

9. Osler W. The growth of truth: as illustrated in the discovery of the circulation of the blood: being the Harveian Oration delivered at the Royal College of Physicians, London, October 18th, 1906. BMJ 1906;2:1077-84.

10. Mythbusters. Myth: People use health system report cards to make decisions about their healthcare. Ottawa: Canadian Foundation for Healthcare Improvement; 2006. Accessible ici : https://www.cfhi-fcass.ca/SearchResultsNews/06-09-01/688b46df -525d-4ba9-98ab-7c1c9b1130d0.aspx (consulté le 21 avril 2020).

11. Burns EM, Pettengell C, Athanasiou T, et al. Understanding the strengths and weaknesses of public reporting of surgeon-specific outcome data. Health Aff (Millwood) 2016;35:415-21.

12. Damani Z, Bohm E, Quan H, et al. Improving the quality of care with a singleentry model of referral for total joint replacement: a preimplementation/ postimplementation evaluation. BMJ Open 2019;9:e028373.

Intérêts concurrents : Danielle Martin est administratrice à l'emploi du Women's College Hospital, à Toronto, Ont. Aucun autre intérêt concurrent n'a été déclaré.

Cet article a été révisé par des pairs.

Affiliations : Women's College Hospital et Département de chirurgie et de politique, gestion et évaluation de la santé (D. Urbach), Université de Toronto; Institut de recherche du Women's College Hospital (D. Urbach); Women's College Hospital et Département de médecine familiale et communautaire (D. Martin), Université de Toronto; Institut pour les solutions au système de santé et les soins virtuels du Women's College Hospital (D. Martin), Toronto, Ont.

Collaborateurs : Les deux auteurs ont substantiellement contribué à l'élaboration et à la rédaction du manuscrit; ils ont rédigé des segments de l'article et ont révisé de façon critique le contenu intellectuel important; ils ont donné leur approbation finale pour la version destinée à être publiée; et ils assument l'entière responsabilité de tous les aspects du travail en veillant à ce que toute question relative à l'exactitude ou à l'intégrité de chaque partie de l'article ait fait l'objet des vérifications et corrections appropriées.

Correspondance : David Urbach, david.urbach@wchospital.ca 